\title{
Sparse Signal Acquisition via Compressed Sensing and Principal Component Analysis
}

\author{
Imrich Andráš ${ }^{1}$, Pavol Dolinský², Linus Michaeli ${ }^{3}$, Ján Šaliga ${ }^{4}$ \\ Technical University of Košice, Faculty of Electrical Engineering and Informatics, Department of Electronics and \\ Multimedia Telecommunications, Letná 9,04200 Košice, Slovakia,imrich.andras@tuke.sk
}

\begin{abstract}
This paper presents a way of acquiring a sparse signal by taking only a limited number of samples; sampling and compression are performed in one step by the analog to information conversion. The signal is recovered with minimal information loss from the reduced data record via compressed sensing reconstruction. Several methods of analog to information conversion are described with focus on numerical complexity and implementation in existing embedded devices. Two novel analog to information conversion methods are proposed, distinctive by their computational simplicity - direct subsampling and subsampling with integration. Proposed sensing methods are intended for and evaluated with real water parameter signals measured by a wireless sensor network. Compressed sensing proves to reduce the data transfer rate by $>80 \%$ with very little signal processing performed at the sensing side and no appreciable distortion of the reconstructed signal.
\end{abstract}

Keywords: Compressed sensing, analog-to-information conversion, sparse signal, signal compression.

\section{INTRODUCTION}

Compressed sensing (CS) [1] appeared in recent years as a promising method of sub-Nyquist sampling, allowing signal acquisition with fewer measurements than previously thought possible. With sparse signals, CS allows for much lower sampling frequencies and lower data transfer rates. The advantage over conventional compression is very little (if any) additional digital signal processing (DSP). The compression is performed directly by the sampling process and most of the overall computational demand is moved to the reconstruction side. This is convenient, for example, in wireless sensor networks (WSN), where available computation power is expensive at sensor nodes, but virtually unlimited at central hubs. Furthermore, certain methods of CS can be implemented on existing devices with no hardware changes required [2]. CS reconstruction is also inherently immune to data loss [3] and the transferred data are naturally encrypted [4]. Since its introduction, CS was tested with WSNs performing temperature [5], EM emission [6], and ECG [7] monitoring, video streaming [8], etc. Other applications of the CS theory include WSN decentralized data storage [9], accelerated MRI [10], or digital modulation recognition [11].

This work was motivated by issues with wireless sensor network (WSN) intended for water quality monitoring of the Danube river [12]. Individual sensor nodes measure multiple water parameter signals (WPS) such as temperature, salinity, dissolved oxygen, $\mathrm{pH}$, etc. [13], [14], [15]. The sensor nodes use solar power for charging the integrated battery. Around $90 \%$ of the WSN node power consumption is drawn by the transmitter [16]. This proved to be of particular concern during winter months, when only a limited amount of solar power is available and the battery performance is reduced due to low temperatures. Significant reduction in power consumption could be achieved by decreasing the amount of data that are being transmitted. The issue with applying conventional (e.g., transform) compression methods is that they require a substantial number of signal samples in order to be effective. With the current sampling period of 1 hour this would mean a very long processing timeframe. The additional computations would also increase the power consumption, countering the power savings gained at the transmitter. These issues can be circumvented by utilizing CS.

This work demonstrates that CS can be used for WPS measurement and efficiently implemented in an existing WSN. Two sampling methods previously not used with CS are proposed, comprising the main novelty of this paper. Section 2 provides an overview of general CS theory and related works. Investigated sampling methods and their properties are discussed in section 3. Experimental results are shown in section 4, where the performance of the proposed methods is evaluated. An optimal CS configuration of WPS monitoring sensor node is proposed at the end of section 4 , followed by the conclusion. 


\section{COMPRESSED SENSING}

The main goal of CS is condensing a signal into a few samples containing all the information, which is referred to as analog-to-information conversion (AIC). This compressed information is transferred to the receiver, where the original signal is reconstructed. No information loss and exact reconstruction is theoretically possible [1].

In order for CS to be applicable, it is assumed that the input signal can be represented by a linear combination of known basis functions. Furthermore, the signal must be sparse, meaning that it consists of only a small number of basic functions. The signal composed of $s$ basis functions is denoted as $s$-sparse. Let us define $L$ basis vectors $\boldsymbol{\psi}_{l} \in \mathbb{R}^{N \times 1}, 1 \leq l \leq L$ to represent any possible input signal components. The columns of the basis matrix $\Psi \in \mathbb{R}^{N \times L}$ consist of these basis vectors. The input signal vector $\mathbf{f} \in \mathbb{R}^{N \times 1}$ can then be described as

$$
\mathbf{f}=\boldsymbol{\Psi} \mathbf{x}
$$

where the $s$-sparse vector $\mathbf{x} \in \mathbb{R}^{L \times 1}$ conducts linear combination [1].

\section{A. Analog-to-Information Conversion}

Conventional ADCs acquire signal samples at equidistant time instants, according to the Nyquist sampling theorem. With signals highly sparse at a certain domain, such sampling means great redundancy in acquired data, since the amount of information contained within a sparse signal is limited. AIC exploits this fact and only takes a limited number of samples, sufficient to represent the information content. The input signal $\mathbf{f} \in \mathbb{R}^{N \times 1}$ is correlated with $M<N$ measurement signals, represented by $M$ rows of the measurement matrix $\boldsymbol{\Phi} \in \mathbb{R}^{M \times N}$. This performs the AIC, with resulting information signal vector

$$
\mathbf{y}=\boldsymbol{\Phi f} \in \mathbb{R}^{M \times 1} .
$$

There are several ways of performing the AIC, either in analog domain, or digitally after conventional sampling [17]. Analog domain implementations allow for overcoming the Nyquist limit of fast ADCs and are used mainly for high bandwidth signals. Digital domain AIC is a computationally efficient alternative to conventional compression methods. Direct digital implementation of (2) is preferable if possible, because it avoids problems associated with analog domain AIC non-idealities. Random modulation and pre-integration (RMPI), random sampling (RS), and random filtering are the most discussed in literature, but other methods may be used for specific applications.

\section{B. Reconstruction}

Let the input signal vector be $s$-sparse on basis $\boldsymbol{\Psi}$, and let bases $\boldsymbol{\Psi}$ and $\boldsymbol{\Phi}$ be incoherent (rows $\boldsymbol{\varphi}_{m}$ of $\boldsymbol{\Phi}$ do not sparsely represent the columns $\boldsymbol{\psi}_{l}$ of $\boldsymbol{\Psi}$, and vice versa). If
$s<M<<N$, the input signal can be reconstructed [18]. An approximation of minimal $M$ needed is given by

$$
M_{\min }=\mu s \log _{10}(N)
$$

assuming zero or negligible noise. $\mu=1$ for incoherent bases, but has to be increased to $\mu=L / N$ if $N<L$. By inserting (1) into (2) the information signal becomes

$$
\mathbf{y}=\boldsymbol{\Phi} \Psi \mathbf{x}
$$

with the reconstruction matrix

$$
\mathbf{A}=\boldsymbol{\Phi} \boldsymbol{\Psi} \in \mathbb{R}^{M \times L} .
$$

At the receiving end of the CS framework, the information signal $\mathbf{y}$, and both bases $\boldsymbol{\Psi}$ and $\boldsymbol{\Phi}$ are known. The only unknown required for reconstruction of (1) is $\mathbf{x}$. Since $\mathbf{A}$ is a rectangular matrix, it cannot be simply inverted and an undetermined system of $M$ equations and $L$ unknowns is to be solved. Here the importance of sparsity is shown, because based on this requirement a unique solution can be found. Out of all the possible solutions, the right solution is the one that is the most sparse [1].

The sparsity $s$ of vector $\mathbf{x}$ can be characterized by its $\ell_{1}$ norm defined as

$$
\ell_{1}:\|\mathbf{x}\|_{1}=\sum_{i=1}^{n}\left|x_{i}\right|
$$

Sparsity is an additional information used to devise the measurement process $\boldsymbol{\Phi}$, and forming a convex optimization problem

$$
\min \|\mathbf{x}\|_{1} \text { subject to } \mathbf{A x}=\mathbf{y}
$$

This guarantees a correct optimal solution, if such exists. The original signal estimate can be found more efficiently as

$$
\hat{\mathbf{f}}=\boldsymbol{\Psi}\left(\mathbf{A}^{T} \mathbf{A}\right)^{-1} \mathbf{A}^{T} \mathbf{y},
$$

where $(*)^{-1}$ denotes the pseudoinverse matrix of $*$. (8) incorporates solving an $\ell_{2}$ minimization problem, but in practical scenarios (7) and (8) yield the same solutions within a noise related uncertainty [19].

The issue with CS is determining the base matrices $\boldsymbol{\Psi}$ and Ф. AIC is performed in such a way that the resulting measurement matrix $\boldsymbol{\Phi}$ shows random attributes, ensuring bases incoherency. The basis matrix $\boldsymbol{\Psi}$ can be based on signal model if the sparse domain is known, e.g. frequency or wavelet. For other signals there is not a specific domain for this purpose, and a suitable dictionary must be learned. Correctly constructed set $\boldsymbol{\Psi}$ of basic functions ensures high compression ratio and low reconstruction error. For WPS basis extraction the principal component analysis (PCA) [20] 
is proposed. PCA is convenient with signals that would require complicated models or their analytical model is unknown. The PCA is particularly prominent in biomedical applications [21]-[24], and has been utilized for basis extraction in various CS applications [3], [7], [10], [25]. The CS framework with both sensing and decoding side is summarized in Fig.1.

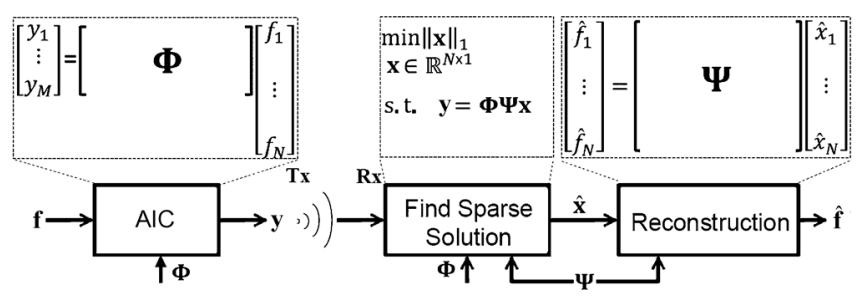

Fig.1. CS framework.

\section{INVESTIGATED MEASUREMENT METHODS AND MATRICES}

\section{A. Random modulation and pre-integration}

The RMPI method of AIC is probably the most studied. A functional diagram of analog RMPI is shown in Fig.2. Input signal $f(t)$ is multiplied by sequence $p(t)$ of \pm 1 's from the random sequence generator (RSG). Output signal of the mixer is being integrated and the integrator output is periodically sampled by a conventional ADC. After each sample is taken, the integrator is reset. Sampling frequency of the $\mathrm{ADC}$ is several times lower than the input signal Nyquist frequency. The only component operating at Nyquist rate is the mixer, any further DSP is performed after compression at a lower rate. The compression itself is performed in the analog domain without DSP. This allows for real-time energy-efficient compression of very high bandwidth signals.

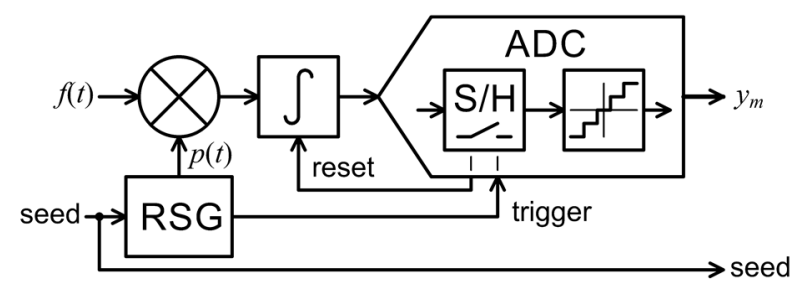

Fig.2. Analog RMPI AIC.

The main disadvantage of analog RMPI is the need for specialized non-ideal analog circuitry. There may be a discrepancy between the expected measurement process $\boldsymbol{\Phi}$ and the actual RMPI behavior [26] resulting in improper reconstruction (8). Accurate modeling of physical circuitry [27] and stochastic phenomena mitigation [28], [29] may be a problem. If the acquired signal is not of excessively high bandwidth, RMPI may be implemented digitally as is shown in Fig.3. The advantage is that the reconstruction error can be significantly lower, since the only non-ideal component is the input ADC. With slow signals like WPS, digital RMPI could be implemented on an existing device solely via firmware upgrade.

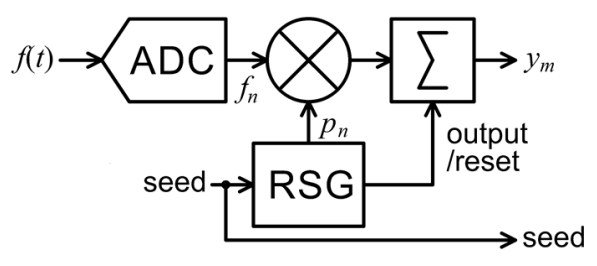

Fig.3. Digital RMPI AIC.

After the input signal is sampled by a Nyquist ADC into its discrete representation $f_{n}$, the signal is multiplied by a random sequence $p_{n}$ of \pm 1 's and summed. The summator is being periodically reset after outputting the accumulated value. All of this DSP runs at Nyquist rate in real time, so the digital RMPI is more suitable for slow signals. Elements $\varphi_{m n}, 1 \leq m \leq M, 1 \leq n \leq N$ of the measurement matrix $\boldsymbol{\Phi} \in \mathbb{R}^{M \times N}$ representing an ideal or digital RMPI can be described as

$$
\varphi_{m n}=\left\{\begin{array}{l}
p_{n},(m-1) C<n \leq m C \\
0
\end{array}\right.
$$

where

$$
C=\frac{N}{M} \in \mathbb{N}
$$

is the compression ratio.

\section{B. Random sampling}

The RS AIC architecture is among the easiest to implement, yet it is rarely studied. RS can be achieved either by triggering an ADC according to RSG (Fig.5.), or simply by discarding samples of a free-running ADC [30]. Both of these approaches are suitable for high bandwidth signals. There is no special analog signal processing and the compression is performed with virtually no DSP. The RS may also be implemented via firmware upgrade on existing devices. Despite all of these advantages the RMPI is by far more common in literature. The reason is probably the limited versatility of RS. RMPI yields low sampling rate, but the input information present between sampling instants is preserved via integration. With RS all of this information is discarded, which practically limits the RS AIC to frequency sparse signals with little expected variance [30].

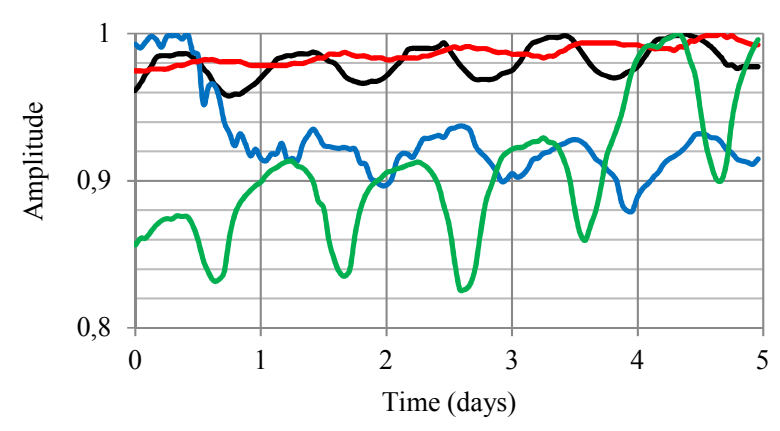

Fig.4. Examples of measured water parameter signals. 
A typical WPS (Fig.4.) contains a large slowly changing DC component, a faster AC component with relatively small amplitude and a small amount of noise [32]. The AC component is a distorted sine wave with basic period of approximately 1 day and a few higher harmonics. The WPS thus carries only limited information content and can be classified as frequency sparse [33], meeting the criteria for RS application.

From all the Nyquist samples of input signal (1) a subset of $M$ samples is selected. $\Xi$ contains indices of selected samples

$$
\Xi=\left\{\xi_{1}, \xi_{2}, \ldots, \xi_{M}\right\}
$$

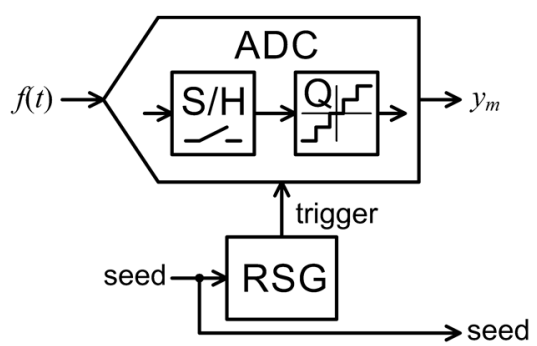

Fig.5. RS AIC.

Entries of $\Xi$ are random numbers, following the rules

$$
\begin{gathered}
\forall m \in \mathbb{N}, 1 \leq m \leq M: \xi_{m} \in \mathbb{N}, 1 \leq \xi_{m} \leq N \\
\forall i, j \in \mathbb{N}, 1 \leq i<j \leq M: \xi_{i}<\xi_{j} .
\end{gathered}
$$

Elements of measurement matrix representing RS AIC can be obtained using (13) as

$$
\varphi_{m n}=\left\{\begin{array}{l}
1, n=\xi_{m} \\
0
\end{array}\right.
$$

and the information signal vector $\mathbf{y}(2)$ elements become

$$
y_{m}=f_{\xi_{m}} \text {. }
$$

\section{Direct subsampling}

Coherent sub-Nyquist sampling without antialiasing filter is not yet covered by CS related literature for an obvious reason. In general, proper reconstruction with such AIC is not possible because of aliasing. However, the basic functions of WPS obtained by PCA are not strictly periodic functions. Elements of $\boldsymbol{\Psi}$ are general waveforms, as is shown in Fig.6.

Only some of the basic functions resemble sinewaves with a period of approximately 1 day. These have a common composition of higher harmonics and interharmonics already incorporated. As such, higher harmonics are not subject to irreversible aliasing. The original signal can be reconstructed provided that the basis harmonic component is sampled at Nyquist rate. Irreversible input information loss occurs if the period of a specific higher harmonic component is exactly equal to the sampling period or its integer multiple.

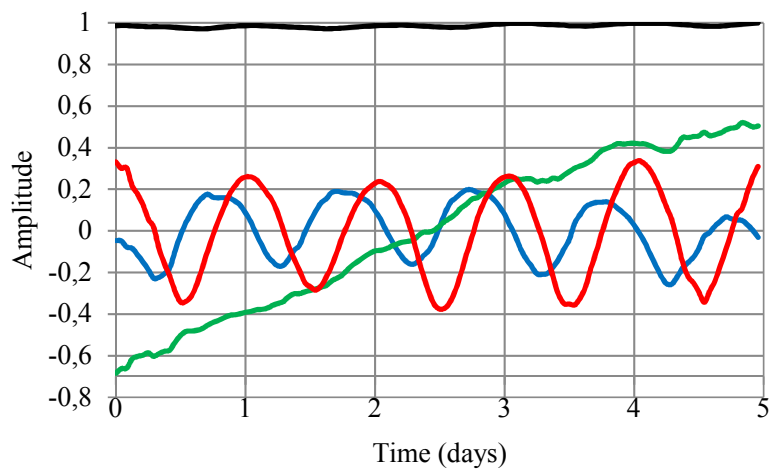

Fig.6. The most significant WPS basis components obtained by PCA.

The presented study considers coherent subsampling via decimation of a Nyquist record, hereafter referred to as direct subsampling (DS). Corresponding measurement matrix consists of elements

$$
\varphi_{m n}=\left\{\begin{array}{l}
1, n=m C \\
0
\end{array},\right.
$$

resulting in information signal (2)

$$
y_{m}=f_{m C} .
$$

DS is proposed as application-specific AIC for WPS because of its simplicity. The implementation on existing devices is even easier than RS, since no RSG is needed. If the existing Nyquist ADC has a fixed clock, samples may be discarded. Better energy efficiency would be achieved if the ADC clock could be reprogrammed to a sub-Nyquist rate, eliminating the need to discard samples.

\section{Subsampling with integration}

Subsampling with integration (SI) is proposed as application-specific simplified alternative to RMPI. The modulator can be omitted because WPS represents absolute values of chemical properties and is non-alternating. Input signal is integrated over the sampling period, as is shown in Fig.7. The integrator is being periodically reset after the integrated signal is sampled.

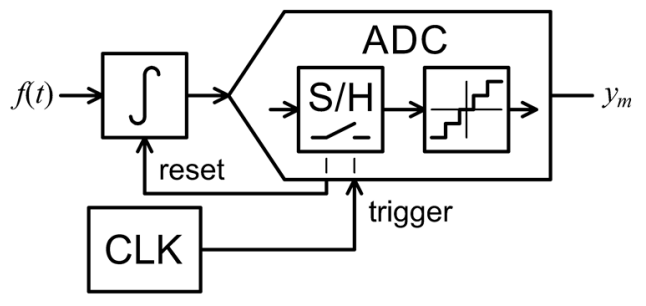

Fig.7. Analog SI AIC. 
Digital implementation of SI AIC as shown in Fig.8. may be preferable due to the reasons discussed in section 3.A. The measurement matrix changes from (11) to

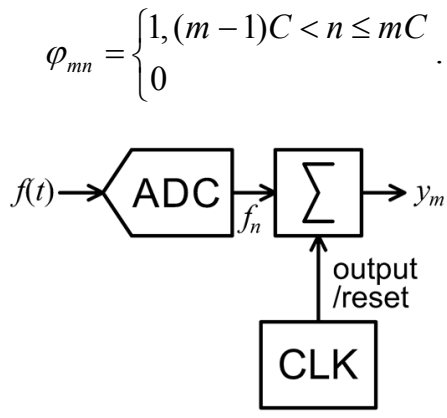

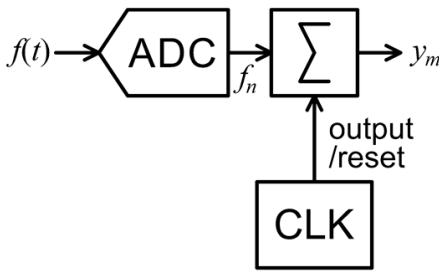

Fig.8. Digital SI AIC.

\section{RESULTS}

Digital implementations of AIC methods described in section 3 were tested using WPS data obtained during the pilot operation of the WSN [13]. All of the tested methods can be implemented as a software add-on on existing sensor nodes. Testing was performed by means of simulations, but it sufficiently represents implementation on the physical WSN.

$L^{\prime}=200$ signal segments with length of $N=120$ (5 days) were randomly chosen from the WPS database and normalized to 1 . This training set was used for extraction of the basis matrix $\boldsymbol{\Psi} \in \mathbb{R}^{N \times L^{\prime}}$ by the PCA. Another set of 200 randomly chosen WPS segments was used for testing. The signal-to-deviation ratio

$$
S D R=10 \log \left(\frac{\sum_{n=1}^{N} \hat{f}_{n}^{2}}{\sum_{n=1}^{N}\left(f_{n}-\hat{f}_{n}\right)^{2}}\right),
$$

where $\hat{f}_{n}$ is the reconstructed signal, was used for evaluation. $S D R$ s of each of the test signals were averaged. With RS and RMPI, $10^{3}$ measurements of each test signal were performed with a new measurement matrix generated every time.

\section{A. Number of principal components used for reconstruction}

Out of all the $L^{\prime}$ extracted basis components, only a limited number has appreciable energy. The rest of the components can be left out of $\boldsymbol{\Psi}$ with little impact on SDR. Achievable $S D R$ with respect to the number of components in model (1) was found by using an identity measurement matrix $\boldsymbol{\Phi}=\mathbf{I}_{N}$ (no resampling) and performing reconstruction (8) with varied $L$.

Achievable $S D R$ increases with greater $L$. For every $L \geq 52$ exact reconstruction is observed with some of the test signals. Exact reconstruction means that $f_{n}=\hat{f}_{n}$ in (19), resulting in average $S D R \rightarrow \infty$ for any $L \geq 52$. Most of the test signals are noiseless, which makes exact reconstruction possible. The behavior of (8) with $L \geq 52$ can be further examined by observing the probability of exact reconstruction (PER), shown in Fig. 10.

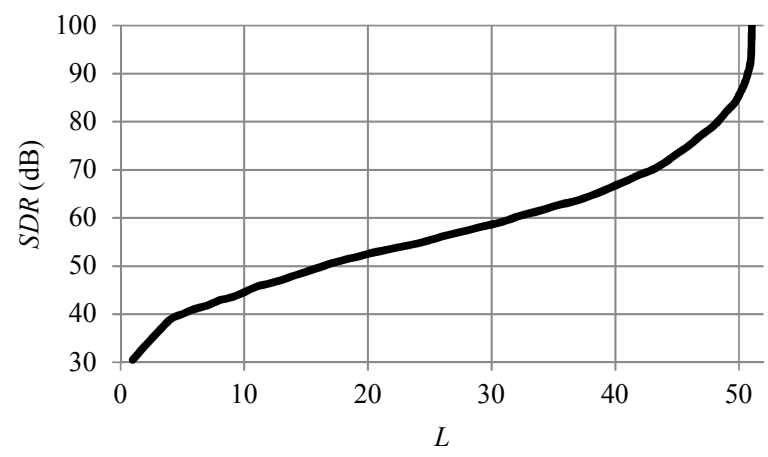

Fig.9. Achievable $S D R$ vs. number of principal components used.

It can be seen that PER is never equal to 1 , since some of the test signals are noisy. Exact reconstruction cannot be expected even with noiseless signals - no training set can be large enough to account for every possible signal variation. With available training set the principal component dictionary $\boldsymbol{\Psi}$ saturates around $L=100$. Increasing the number of principal components further brings no appreciable improvement.

Based on results shown in Fig.9. and Fig.10., the number of principal components used was chosen to be $L=50$, which applies to all further evaluations. $L<200$ was chosen on purpose in order to simulate an unsaturated principal component dictionary expected in practical applications. Smaller $L$ also results in faster reconstruction due to smaller size of matrices while having no impact on AIC.

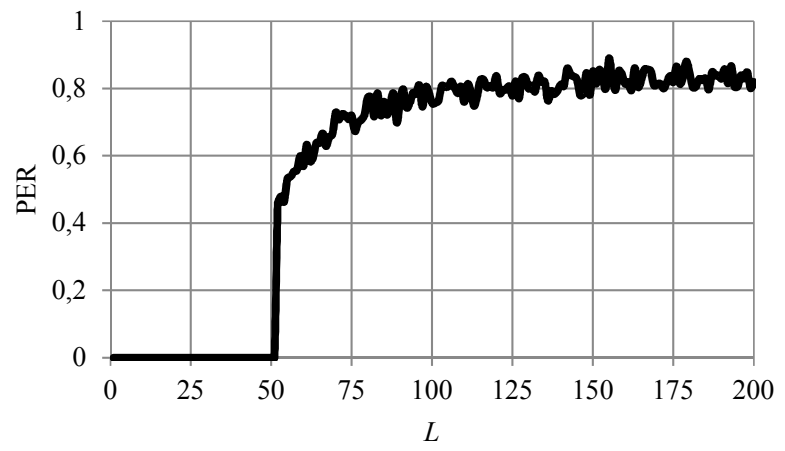

Fig.10. PER vs. number of principal components used.

\section{B. Required sampling rate}

The actual threshold of $M$ required for successful reconstruction is a subject of debates. Analytical derivations such as (3) have been presented, but their use may be difficult in practice. The actual signal sparsity is unknown in advance, particularly with numerically learned [20] bases and signals degraded by random and quantization noise. 


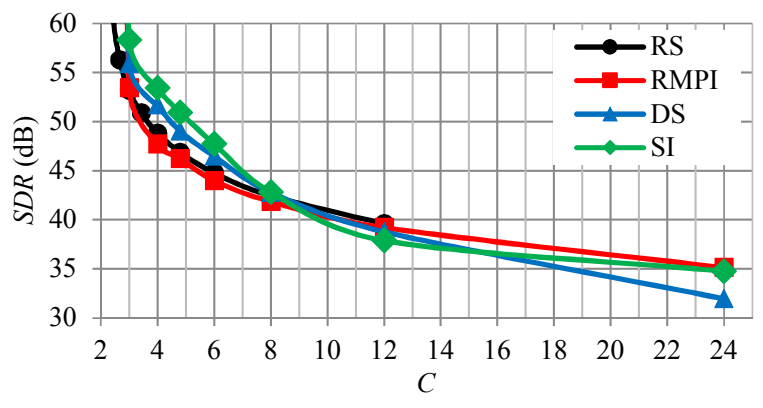

Fig.11. $S D R$ vs. compression ratio.

Any analytical derivation of $M_{\text {min }}$ is probabilistic. Choosing $M<M_{\min }$ does not mean that the reconstruction is impossible, but rather that there is a high risk of picking particular signal values that result in unsuccessful signal recovery. The most reliable way of choosing an appropriate $M$ for certain application is an experiment, where $M$ is varied and reconstruction error is observed. Unknown $s$ on a particular $\boldsymbol{\Psi}$ base can be determined retroactively by observing $\mathbf{x}$ in successful reconstructions. $S D R$ as a function of compression ratio (12) for investigated AIC methods is shown in Fig.11.

The performance of all AIC methods is similar within approximately $6 \mathrm{~dB} S D R$ range. SI achieves the highest $S D R$ at both small and high compression ratios, while RS and RMPI perform slightly better in the intermediate range. DS may be preferable due to its simplicity, despite worse performance at high $C$. The final choice of AIC method implemented in WSN would strongly depend on the required SDR.

\section{Resistance to quantization}

High ADC resolution, denoted in number of bits $(N O B)$, is easily achievable with signals as slow as WPS. However, the total amount of data payload that is to be transferred is proportional to $N O B$. Lower $N O B$ is beneficial for overall sensor node power consumption, and can be achieved by rescaling existing high-resolution samples. Influence of quantization on $S D R$ was tested using a simulated ideal unipolar $\mathrm{ADC}$ with a range of 2. Results for $C=6$ and varying resolution are shown in Fig. 12.

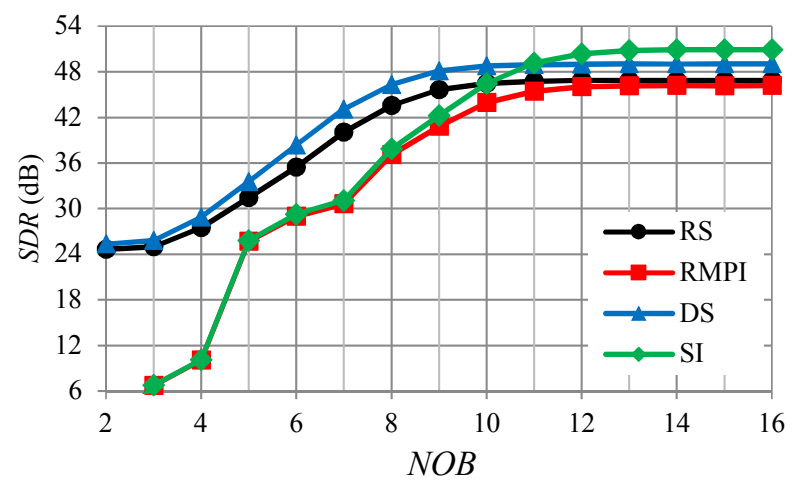

Fig.12. $S D R$ vs. ADC resolution.
RS and DS show slightly greater robustness against quantization than RMPI and SI. The highest $S D R$ is achievable by SI, which is consistent with results from the previous section. Increasing $N O B$ above 12 yields virtually no improvement in $S D R$ with all evaluated AIC methods.

\section{Requirements on the reconstruction error}

Results presented above show that the SDR is dependent on compression ratio and quantization noise. The specific combination of $C$ and $N O B$ defines the power consumption, which is in trade-off with the SDR. How high the $S D R$ should be, and whether the introduced misinformation is of any concern, remains to be decided by the end user. Experiments show that $S D R$ above $30 \mathrm{~dB}$ is enough to capture the slowly changing DC component of any available WPS. In order to preserve the smallest AC components present in the database, $S D R$ should remain above $50 \mathrm{~dB}$.

The authors propose to use SI AIC with $C=6$ and $N O B=12$. The power consumption with such configuration would be reduced by approximately $80 \%$ while maintaining $S D R \geq 50 \mathrm{~dB}$. An example of input and reconstructed signal obtained with this configuration and $S D R=51.43 \mathrm{~dB}$ is shown in Fig.13. Further reduction of power consumption is possible at the cost of lower $S D R$.

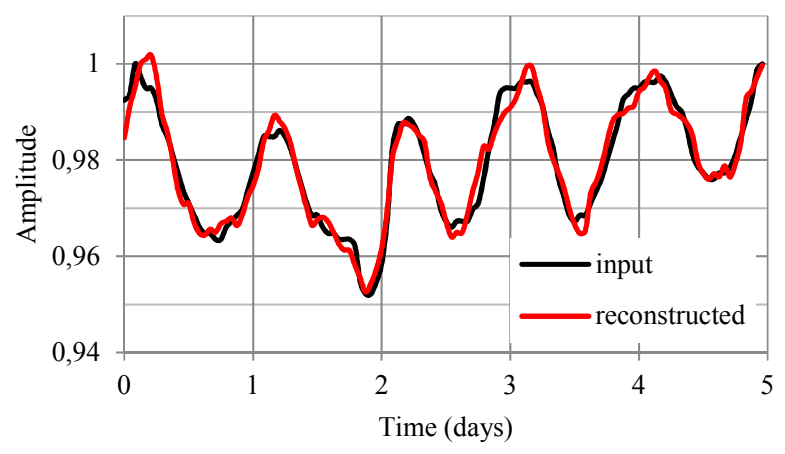

Fig.13. Example of input and reconstructed signal, SI method, $C=6, N O B=12, S D R=51.43 \mathrm{~dB}$.

\section{CONCLUSION}

CS was originally promoted as near-optimal and lossless on orthonormal bases. However, practical WPS results show that the reconstruction is not exact if appreciable compression ratio is achieved. This was also concluded in some of the cited works on other CS applications, e.g. ECG [7]. Considering the reasonable compression at minimal computation effort, CS is still a reasonable choice for WPS monitoring WSN. RS and RMPI methods of AIC have been previously used in WSNs and proved to be efficient also with WPS. The presented DS and SI methods pose even lower computational demand on the sensor node. The SI method at the same time outperforms previously presented AIC methods in achievable SDR. AIC configuration that would reduce the sensor node power consumption by approximately $80 \%$ was proposed and remains to be implemented on existing WSN nodes. 


\section{ACKNOWLEDGMENT}

The work is a part of the project supported by the Science Grant Agency of the Slovak Republic (No. 1/0722/18).

\section{REFERENCES}

[1] Donoho, D.L. (2006). Compressed sensing. IEEE Transactions on Information Theory, 52 (4), 12891306.

[2] Andráš, I., Dolinský, P., Michaeli, L., Šaliga, J. (2018). A time domain reconstruction method of randomly sampled frequency sparse signal. Measurement, 127, 68-77.

[3] Wu, L., Yu, K., Hu, Y., Wang, Z. (2014). CS-based framework for sparse signal transmission over lossy link. In IEEE International Conference on Mobile Ad Hoc and Sensor Systems, Philadelphia, USA. IEEE, 680-685.

[4] Liu, H., Xiao, D., Zhang, R., Zhang., Y., Bai, S. (2016). Robust and hierarchical watermarking of encrypted images based on Compressive Sensing. Signal Processing: Image Communication, 46, 41-51.

[5] Xiao, X., He, Q., Fu, Z., Xu, M., Zhang, X. (2016). Applying CS and WSN methods for improving efficiency of frozen and chilled aquatic products monitoring system in cold chain logistics. Food Control, 60, 656-666.

[6] Bellan, D., Pignari, S.A. (2015). Monitoring of electromagnetic environment along high-speed railway lines based on compressive sensing. Progress in Electromagnetics Research C, 58, 183-191.

[7] Craven, D., McGinley, B., Kilmartin, L., Glavin, M., Jones, E. (2016). Energy-efficient Compressed Sensing for ambulatory ECG monitoring. Computers in Biology and Medicine, 71, 1-13.

[8] Angayarkanni, V., Radha, S. (2016). Design of bandwidth efficient compressed sensing based prediction measurement encoder for video transmission in wireless sensor networks. Wireless Personal Communications, 87, 1-21.

[9] Talari, A., Rahnavard, N. (2016). CStorage: Decentralized compressive data storage in wireless sensor networks. Ad Hoc Networks, 37, 475-485.

[10] Zong, F., Eurydice, M.N., Galvosas, P. (2016). Fast reconstruction of highly undersampled MR images using one and two dimensional principal component analysis. Magnetic Resonance Imaging, 34, 227-238.

[11] Sun, Z., Wang, S., Chen, X. (2016). Feature-based digital modulation recognition using compressive sampling. Mobile Information Systems, 10, 9754162.

[12] Maceková, L., Žiga, M. (2014). The wireless sensor network concept for measurement of water quality in water streams. Acta Electrotechnica et Informatica, 14 (2), 60-67.

[13] Šaliga, J., Žiga, M., Galajda, P., Drutarovský, M., Kocur, D., Maceková, L. (2015). Wireless sensor network for river water quality monitoring. In $X X I$ IMEKO World Congress "Measurement in Research and Industry”, Prague, Czech Republic. IMEKO, 17451750 .
[14] Galajda, P., Drutarovský, M., Šaliga, J., Žiga, M., Maceková, L., Marchevský, S., Kocur, D. (2015). Sensor node for the remote river quality monitoring. In MEASUREMENT 2015. Bratislava, Slovakia: IMS SAS, 313-316.

[15] Šaliga, J., Kocur, D., Galajda, P., Drutarovsky, M., Macekova, L., Andráš, I., Michaeli, L. (2017). Multiparametric sensor network for water quality monitoring. In IMEKO TC19 Workshop on Metrology for the Sea, Naples, Italy. IMEKO, 123-126.

[16] Stojmenović, I. (2005). Energy scavenging and nontraditional power sources for wireless sensor networks. In Handbook of Sensor Networks: Algorithms and Architectures. John Wiley \& Sons, 75-106.

[17] Daponte, P., De Vito, L., Rapuano, S., Tudosa, I. (2017). Analog-to-information converters in the wideband RF measurement for aerospace applications: Current situation and perspectives. IEEE Instrumentation \& Measurement magazine, 20 (1), 2028.

[18] Candes, E.J., Wakin, M.B. (2008). An introduction to compressive sampling. IEEE Signal Processing Magazine, 25 (2), 21-30.

[19] Fung, G., Mangasarian, O.L. (2011). Equivalence of minimal $\ell_{0}$ and $\ell_{p}$-norm solutions of linear equalities, inequalities and linear programs for sufficiently small p. Journal of Optimization Theory and Applications, 151 (1), 1-10.

[20] Palese, L.L. (2018). A random version of principal component analysis in data clustering. Computational Biology and Chemistry, 73, 57-64.

[21] Phyniomark, A., Hu, H., Phukpattaranont, P., Limsakul, C. (2012). Application of linear discriminant analysis in dimensionality reduction for hand motion classification. Measurement Science Review, 12 (3), 8289.

[22] Ji, Y., Sun, S., Xie, H.B. (2017). Stationary waveletbased two-directional two-dimensional principal component analysis for EMG signal classification. Measurement Science Review, 17 (3), 117-124.

[23] Oesterlein, T.G., Lenis, G., Luik, A., Verma, B., Schmitt, C., Dossel, O. (2014). Removing ventricular far field artifacts in intracardiac electrograms during stable atrial flutter using the periodic component analysis - proof of concept study. In Electrocardiology 2014: Proceedings of 41th International Congress on Electrocardiology. Bratislava, Slovakia: IMS SAS, 4952.

[24] Roštáková, Z., Rosipal, R. (2018). Time alignment as a necessary step in the analysis of sleep probabilistic curves. Measurement Science Review, 18 (1), 1-6.

[25] Huang, H., Ouyang, H., Gao, H., Guo, L., Li, D., Wen, J. (2016). A feature extraction method for vibration signal of bearing incipient degradation. Measurement Science Review, 16 (3), 149-159.

[26] Abari, O., Lim, F., Chen, F., Stojanović, V. (2013). Why analog-to-information converters suffer in highbandwidth sparse signal applications. IEEE Transactions on Cirsuits and Systems - I: Regular Papers, 60 (9), 2273-2284. 
[27] Daponte, P., De Vito, L., Iadarola, G., Iovini, M., Rapuano, S. (2016). Experimental comparison of two mathematical models for Analog-to-Information Converters. In $21^{\text {st }}$ IMEKO TC4 Symposium "Measurements of Electrical Quantities 2016" (and 19th International Workshop on ADC and DCA Modelling and Testing, IWADC): Understanding the World Through Electrical and Electronic Measurement, Budapest, Hungary. IMEKO, 65-70.

[28] Daponte, P., De Vito, L., Iadarola, G., Rapuano, S. (2016). PRBS non-idealities affecting Random Demodulation Analog-to-Information Converters. In $21^{\text {st }}$ IMEKO TC4 Symposium "Measurements of Electrical Quantities 2016" (and 19th International Workshop on ADC and DCA Modelling and Testing, IWADC): Understanding the World Through Electrical and Electronic Measurement, Budapest, Hungary. IMEKO, 71-60.

[29] Daponte, P., De Vito, L., Iadarola, G., Rapuano, S. (2016). Effects of PRBS jitter on random demodulation analog-to-information converters. In IEEE Metrology for Aerospace, Florence, Italy. IEEE, 630-635.
[30] Candes, E., Becker, S. (2013). Compressive sensing: Principles and Hardware implementations. In ESSCIRC 2013: $39^{\text {th }}$ European Solid - State Circuits Conference, Bucharest, Romania. IEEE, 22-23.

[31] Wakin, M., Becker, S., Nakamura, E., Grant, M., Sovero, E., Ching, D., Yoo, J. (2012). A non-uniform sampler for wideband spectrally-sparse environments. IEEE Journal on Emerging and Selected Topics in Circuits and Systems, 2 (3), 516-529.

[32] Faculty of Electrical Engineering and Informatics, Technical University of Košice, Slovakia. (2013-2015). WSN-AQUA Wireless Sensor Network for Water Quality Monitoring (project).

[33] Lopes, M.E. (2013). Estimating unknown sparsity in compressed sensing. In $30^{\text {th }}$ International Conference on Machine Learning (ICML 2013), Atlanta, Georgia, USA. International Machine Learning Society (IMLS), 1254-1262.

Received April 13, 2018 Accepted September 03, 2018 